\title{
Linx
}

Revue des linguistes de l'université Paris X Nanterre

$82 \mid 2021$

Entre vieillissement et innovation : le changement linguistique

\section{Présentation du numéro}

\section{Gaétane Dostie, Sascha Diwersy et Agnès Steuckardt}

\section{OpenEdition}

Journals

Édition électronique

URL : https://journals.openedition.org/linx/8005

DOI : $10.4000 / \operatorname{linx} .8005$

ISSN : 2118-9692

Éditeur

Presses universitaires de Paris Nanterre

Référence électronique

Gaétane Dostie, Sascha Diwersy et Agnès Steuckardt, « Présentation du numéro », Linx [En ligne], 82 | 2021, mis en ligne le 15 juillet 2021, consulté le 20 juillet 2021. URL : http://journals.openedition.org/ linx/8005; DOI : https://doi.org/10.4000/linx.8005

Ce document a été généré automatiquement le 20 juillet 2021.

Département de Sciences du langage, Université Paris Ouest 


\title{
Présentation du numéro
}

\author{
Gaétane Dostie, Sascha Diwersy et Agnès Steuckardt
}

A la mémoire de Jean-François Sablayrolles

\section{Problématique}

1 Les problématiques croisées du vieillissement, de l'innovation et du changement linguistiques interpellent les locuteurs et les descripteurs de la langue depuis longtemps : ainsi Horace comparait-il le devenir des mots au cycle des générations ${ }^{1}$. Pour nous en tenir à l'âge de la linguistique, citons Bréal, qui s'intéresse en 1883 à ce qu'il nomme des lois "qui président à la transformation des sens, au choix d'expressions nouvelles, à la naissance et à la mort des locutions » (p. 133). Il critiquera cependant lui-même cette métaphore biologique, développée au XIX siècle, sous l'influence du darwinisme; selon Schleicher par exemple, "les langues sont des organismes naturels qui, en dehors de la volonté humaine et suivant des lois déterminées, naissent, croissent, se développent, vieillissent et meurent » $(1868$, p. 3). La vie des mots, publié en 1887 par Darmesteter, reprend l'analogie, que Bréal entreprend alors de déconstruire: "dire que les mots naissent, vivent entre eux et meurent, cela est, n'est-il point vrai ? pure métaphore. Parler de la vie du langage, appeler les langues des organismes vivants, c'est user de figures qui peuvent servir à nous faire mieux comprendre, mais qui, si nous les prenions à la lettre, nous transporteraient en plein rêve. M. Darmesteter ne s'est peut-être pas toujours assez défié de cette sorte de mise en scène » ([1887] 1897, p. 306).

2 Dans Essai de sémantique (1897), Bréal examine en termes plus rigoureusement linguistiques une série de phénomènes, très divers, en lien avec les thématiques précitées. Parmi les phénomènes étudiés, certains seraient des "causes » ou, en tout cas, des «déclencheurs» du changement linguistique (par exemple la tendance à la spécialisation dans un fonctionnement grammatical, dénommée ultérieurement grammaticalisation par Meillet 1912 [1965]); d'autres seraient plutôt des effets ou des conséquences du changement. En guise d'exemple, citons la polysémie, qui peut 
notamment résulter d'une métaphorisation ou d'une métonymisation, mais aussi de ce que Bréal appelle l'« élargissement » de sens ou encore le " rétrécissement » de sens. De même, l'auteur envisage l'existence momentanée d'une synonymie exacte entre mots, découlant de changements survenus au sein d'un système linguistique. Dans un tel cas, la synonymie serait toutefois rapidement éradiquée du fait que les locuteurs auraient spontanément tendance à "répartir » les doublons, c'est-à-dire à les différencier au plan conceptuel ou, simplement, à éliminer l'un d'entre eux.

3 Les travaux pionniers de Bréal n'ont pas réponse à tout. Un siècle plus tard, on cherche toujours à modéliser le vieillissement, l'innovation et le changement linguistiques, à en dégager les causes et à en mesurer les effets sur l'organisation synchronique des systèmes langagiers. Dans cette perspective, Croft (2000) élabore la Théorie de la sélection de l'énoncé, inspirée de la théorie généralisée de la sélection développée par le philosophe des sciences D. Hull (Evans et Green, 2006). On reconnaît ici l'ancienne et contestée métaphore biologique où interviennent les concepts de 'réplicateur' (correspondant à un item linguistique quelconque utilisé dans un énoncé, nommé lingueme), d'interacteurs' (désignant les locuteurs/interlocuteurs), de 'réplication' (qui serait soit "normale », soit « altérée »), de 'sélection' (qui amènerait à reproduire une réplication « altérée » et à la propager) et, enfin, de 'lignée' (en écho à l'étymologie). Ce modèle, dont la pertinence reste à interroger, peine à rendre compte du changement linguistique et des phénomènes connexes: tout au plus décrit-il la manière dont un changement donné pourrait s'enraciner en langue. Les auteurs assortissent donc leur théorie de la sélection de l'énoncé d'un certain nombre de maximes interactionnelles qui guideraient le comportement langagier et amèneraient les locuteurs à opter soit pour une réplication normale (par exemple 'Parlez de manière telle qu'on vous comprenne'), soit pour une réplication altérée - au cœur du changement (par exemple 'Parlez de manière drôle, amusante' ou encore 'Parlez de sorte qu'on vous remarque').

4 Le respect des maximes mentionnées ci-dessus serait intentionnel. Or une réplication altérée n'est pas forcément intentionnelle - notamment lorsqu'il y a grammaticalisation ou encore lorsqu'un changement phonétique conditionné se produit - de sorte que le modèle introduit plus haut ne parvient pas à couvrir tous les cas de figure inhérents au changement et aux phénomènes afférents, tels le vieillissement et l'innovation. Aussi, une modélisation en symbiose, qui ferait intervenir des procédés misant sur l'existence de changements tant inconscients que conscients, paraît incontournable.

\section{Contributions au numéro}

5 Ce numéro réunit onze articles dans lesquels les problématiques croisées du vieillissement, de l'innovation et du changement linguistiques sont scrutées, principalement à travers des études de cas portant sur le français et, de manière complémentaire, sur quelques langues d'Asie et d'Afrique. Ces articles s'attachent à des phénomènes perceptibles dans une diachronie courte, qui relèvent des niveaux lexical, grammatical et phonétique.

6 Six études sont consacrées au lexique, terrain traditionnellement privilégié pour l'observation du changement linguistique. Les deux premières proposent une réflexion générale sur le changement lexical, les quatre autres se consacrant à des études de cas. 
Dans le texte liminaire, Jean-François Sablayrolles attire notre attention sur le curieux mélange de générations qui règne dans la synchronie d'un discours: quand nous parlons, nous utilisons des mots d'époques variées. Avons-nous conscience de cette hétérogénéité diachronique ? Jean-François Sablayrolles nous invite à nous interroger sur la perception de l'archaïsme et sur le sentiment de néologicité.

7 L'article d'olga Galatanu aborde, quant à lui, la question de savoir comment le concept du cinétisme discursif et sémantique s'articule avec la théorisation du changement linguistique. Partant du cadre théorique de la Sémantique des Possibles Argumentatifs (SPA), l'auteure retrace, à titre d'illustration, les mouvements de vieillissement et d'innovation simultanés que connaissent respectivement les noyaux de signification des mots réclame et publicité tels qu'ils se manifestent à travers leurs occurrences dans le corpus Frantext.

Abordant la question du changement sous l'angle de la terminologie, Pascale Dury illustre pour sa part le cas de l'abandon pur et simple de lexèmes dans les domaines de spécialité. Elle constate par exemple, dans un corpus de cinq grandes revues de pharmacologie compilées de 1993 à 2017, la disparition de composés en micro- (tels que microglobule, microvessel, microtissue), auxquels sont préférés les composés en nano-, en conformité avec les évolutions de la chimie au XXI ${ }^{\mathrm{e}}$ siècle.

9 Si le vocabulaire de spécialité permet d'observer, en quelque sorte in vitro, des phénomènes extrêmes, la disparition n'est généralement pas, dans la langue commune, aussi radicale. Elle s'opère progressivement par ce processus d'obsolescence qui n'est pas l'exact symétrique du processus d'émergence: alors que, pour peu que la documentation le permette, il est possible de donner une date de première attestation et une période de montée en fréquence d'un mot, rares sont les cas où l'on parvient à déterminer le moment précis de la disparition, notamment parce que la disparition n'est pas totale ou universelle (Steuckardt, 2015). Ainsi la forme asteure, étudiée par Cristina Petraș, a disparu en français de France, mais non dans les français nordaméricains. D'après la base Frantext, cette agglutination de à cette heure, formée en moyen français, est courante en français classique, mais sa fréquence décroît par la suite. Si elle est en français de France un exemple d'obsolescence, elle fonctionne au contraire dans les français nord-américains comme un marqueur discursif dynamique, utilisé à la manière de maintenant.

10 Les deux contributions suivantes abordent la question de la diachronie lexicale par l'analyse outillée de corpus journalistiques contemporains. S'appuyant sur un corpus de presse française, Sascha Diwersy, Agata Jackiewicz, Giancarlo Luxardo et Agnès Steuckardt mènent une enquête de sémantique historique sur le mot numérique: ils suivent, à travers les articles du Monde, l'émergence d'un sens nouveau, montrent comment la relation au nombre, directe dans les premiers emplois de l'adjectif (calcul numérique), devient beaucoup plus lointaine dans son utilisation récente, technologique (téléphone numérique), puis sociale (fracture numérique). Leur analyse invite à se demander si l'empilement de strates discursives, dans un laps de temps relativement bref, n'est pas susceptible d'opacifier le signe.

11 Alors que la contribution de Diwersy et al. utilise le discours pour comprendre le lexique, celle de Marie Chandelier s'appuie sur la connaissance du lexique pour analyser le discours. Cette contribution montre comment le discours joue avec l'épaisseur temporelle du lexique. L'auteure analyse comment les tours du type la bête du Gévaudan, le monstre des Ardennes, le loup garou de Dôle, typiques d'une ancienne presse 
à sensation, sont repris dans la presse contemporaine, par des tours comme la bête des Vosges, pour réveiller des peurs ancestrales. Maintenant des lexèmes et des tours anciens en usage, l'archaïsme lexical permet la résurgence d'un univers référentiel passé et exploite dans l'usage discursif la plasticité diachronique du lexique. québécois que suivent Gaétane Dostie ainsi que Sophie Piron et Nadine Vincent. La contribution de Gaétane Dostie retrace l'histoire de la concurrence synonymique entre les périphrases à valeur progressive être à + infinitif, être après + infinitif et être en train de + infinitif en français québécois de la fin du XIX ${ }^{\mathrm{e}}$ siècle à nos jours. Comment la dernière venue a-t-elle supplanté les deux autres? Gaétane Dostie explore les bases de données Canadiana, BAnQ et Eureka.cc pour mettre en évidence leurs trajectoires respectives. Alors qu'à la fin du XIX être à + infinitif, hérité du français de la colonie, est la forme normée la plus courante, être en train de + infinitif semble "s'être faufilée » par le biais en particulier de la diffusion écrite des français d'Europe. Quant à être après + infinitif, elle était encore bien présente dans l'oral spontané au cours des années 1970, mais elle recule désormais derrière être en train de + infinitif. Pour expliquer ce succès, Gaétane Dostie invoque notamment la polyvalence sémantique de être en train de + infinitif, qui peut marquer une progressivité focalisée, mais aussi une progressivité durative.

Pour leur part, Sophie Piron et Nadine Vincent se penchent sur l'évolution de l'alternance, restée depuis toujours quelque peu énigmatique, des modes indicatif et subjonctif dans les subordonnées introduites par la conjonction après que. Pour ce faire, les auteures dressent un bilan exhaustif du traitement dont a fait l'objet cette alternance de la part des grammaires et des dictionnaires de référence du français 
depuis le $\mathrm{XVI}^{\mathrm{e}}$ siècle pour aboutir à la description récente proposée par le dictionnaire en ligne du français québécois contemporain Usito, ouvrant ainsi la perspective vers l'articulation des dimensions de variation diachronique et diatopique.

17 Le dernier article examine le cas emblématique de la diphtongue -oi. Hugo SaintAmand Lamy mène l'enquête sur la variante antérieure de la diphtongue -oi (p. ex. poire [pwEs]), ainsi que sur le maintien d'un contraste phonémique entre /e/ et $/ \varepsilon /$ devant /

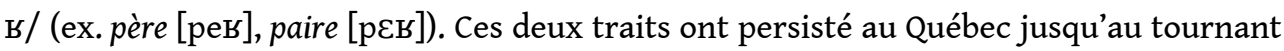
$\mathrm{du} \mathrm{XXI}{ }^{\mathrm{e}}$ siècle, ce qui peut permettre, mutatis mutandis, de mieux comprendre le déclin survenu dans le français de France à l'âge classique. S'appuyant sur 152 témoins québécois nés entre 1872 et 1997, Hugo Saint-Amand Lamy montre qu'au XVII ${ }^{\mathrm{e}}$ siècle en France, comme au XIX siècle au Québec, c'est la variante de prestige qui l'a emporté.

S'il arrive que certains traits phonétiques et linguistiques disparaissent complètement, la situation est habituellement plus complexe : anciens et nouveaux usages coexistent à différents niveaux et degrés, laissant aux locuteurs le soin de régler le jeu inhérent à la nature diachronique de la langue.

\section{BIBLIOGRAPHIE}

BRÉAL, M., 1883, « Les lois intellectuelles du langage. Fragment de sémantique », Annuaire de l'association des études grecques en France, 17, p. 132-142.

BRÉAL, M., 1897, Essai de sémantique. Sciences de significations, Paris, Hachette.

CROFT, W., 2000, Explaining Language Change: An Evolutionary Approach, London, Longman.

EVANS, V. et M. GREEN, 2006, Cognitive Linguistics. An Introduction, Mahwah, Lawrence Erlbaum Associates et Edimburgh University Press.

MEILLET, A., 1912 [1965], « L'évolution des formes grammaticales », Linguistique historique et linguistique générale, Paris, Honoré Champion, p. 130-148.

SCHLEICHER, A., 1868, La théorie de Darwin et la science du langage. De l'importance du langage pour l'histoire naturelle de l'homme, traduit de l'allemand par Pommayrol, Paris, Librairie A. Franck.

STEUCKARDT, A., 2015, « Révolution et disparition. Parcours de quelques ci-devant désignants socio-politiques ", Claire Badiou-Monferran, Thomas Verjans (éds), Disparitions. Contributions à l'étude du changement linguistique, Paris, Champion, p. 191-200.

\section{NOTES}

1. "Ita verborum vetus interit aetas / Et juvenum ritu florent modo nata, vigentque » (ainsi meurent les vieilles générations de mots, et les nouvelles, comme des jeunes gens, s'épanouissent et prennent force » (Art poétique, trad. F. Richard, Paris, Garnier, 1944, I, 61-62). 
AUTEURS

GAÉTANE DOSTIE

Université de Sherbrooke

SASCHA DIWERSY

Université Paul Valéry Montpellier 3

\section{AGNÈS STEUCKARDT}

Université Paul Valéry Montpellier 3 